\title{
Highly sensitive MEMS frequency modulated accelerometer with small footprint
}

\author{
Eurico Esteves Moreira $^{\mathrm{a}, \mathrm{b}, *}$, Burkhard Kuhlmann ${ }^{\mathrm{c}}$, Filipe Serra Alves ${ }^{\mathrm{b}}$, Rosana Alves Dias ${ }^{\mathrm{b}}$ \\ , Jorge Cabral ${ }^{\mathrm{a}}$, João Gaspar ${ }^{\mathrm{b}}$, Luis Alexandre Rocha ${ }^{\mathrm{a}, \mathrm{b}}$ \\ a Algoritmi Center, University of Minho, 4800-058, Guimarães, Portugal \\ b International Iberian Nanotechnology Laboratory (INL), 4715-330, Braga, Portugal \\ c Automotive Electronics Group, Robert Bosch GmbH, 72762, Reutlingen, Germany
}

\section{A R T I C L E I N F O}

\section{Article history:}

Received 24 November 2019

Received in revised form 17 March 2020

Accepted 7 April 2020

Available online 15 April 2020

\section{Keywords:}

Accelerometer

Double-ended tuning fork (DETF)

resonators

Frequency modulated

Micro-lever mechanism

Microelectromechanical systems (MEMS)

\begin{abstract}
A B S T R A C T
A single-axis resonant MEMS accelerometer is presented here. The goal is to achieve the maximum sensitivity on a set of predefined constraints: small footprint of $500 \mu \mathrm{m} \times 500 \mu \mathrm{m}$, vacuum operation under $150 \mathrm{~Pa}$ (requirement for a single-chip IMU) and fabrication using a Bosch silicon surface micromachining process. The sensor is composed by double-ended tuning fork resonators in differential architecture and a force amplification mechanism to increase its sensitivity. A complete characterization of the device was performed including closed-loop operation. A proportional-integral-derivative closed-loop controller architecture updates in real-time the excitation frequency to half the resonance frequency of the resonators. A scale-factor of $170 \mathrm{~Hz} / \mathrm{g}$ and a non-linearity of $0.63 \% \mathrm{FS}$ (operation range of $\pm 1 \mathrm{~g}$ ) were experimentally measured. The relative sensitivities of $0.08 \% \mathrm{~Hz} / \mathrm{g} / \mathrm{nkg}$ and $0.48 \% \mathrm{~Hz} / \mathrm{g}$ are among the highest reported for DETF-based devices. Long-term $(700 \mu \mathrm{g} / \sqrt{ } \mathrm{Hz}$ noise floor measured), dynamic and thermal drift measurements are also reported. The differential operation improved the thermal performance by $77 \%$.
\end{abstract}

(c) 2020 Elsevier B.V. All rights reserved.

\section{Introduction}

Microelectromechanical systems (MEMS) accelerometers have found applications in several markets, from the consumer or automotive electronics to space applications [1], due to their small dimensions, low-weight, reduced production costs (mass production) and low energy consumption [2-4]. However, the constant need for better performance, lower production costs and higher integrability is still driving improvements on the accelerometer state-of-the-art and the seek for alternatives to the well-known open-loop capacitive approaches. The integration of several sensors in the same multi-axis inertial measurement unit (IMU), has been offered by leading semiconductor companies [5], in singlechip format, but with the different sensors fabricated separately (separate dies) and encapsulated at different pressures. A singledie and single-process IMU would require encapsulation of all the sensors (gyroscopes, accelerometers and magnetometers) under the same capsule, at the same pressure [6,7]. This would result

\footnotetext{
* Corresponding author at: Algoritmi Center, University of Minho, 4800-058, Guimarães, Portugal.

E-mail address: id5974@alunos.uminho.pt (E. Esteves Moreira).
}

in a great advantage in terms of cost, size reduction, ease of fabrication and packaging of IMUs, especially in the automotive and consumer electronics industries. While, gyroscopes are generally encapsulated in vacuum because of the need of high-quality factors, open-loop capacitive accelerometers being sensitive to external vibrations (lack of vibration robustness), require more effort for closed-loop operation at low pressure [7]. Two alternatives can be used to address this limitation: the use of closed-loop approaches to keep the proof-mass at the central position using mechanisms such as sigma-delta [8], or frequency modulated devices [5,9-11]. Resonant MEMS offer as main advantages a high dynamic range (no displacement limitation), immunity to pull-in and a quasi-digital output $[9,11]$. Frequency modulation is well-established and several devices have been recently reported: accelerometers (in-plane $[3,10]$ and out-of-plane [12]), inclinometers [4], pitch gyroscopes [13], magnetometers [6,14] and axial strain sensors [15]. Regarding the accelerometers developed, mainly two types of resonators were used, single beam $[10,11]$ and double-ended tuning forks (DETF) $[3,16]$. These are sensitive to stress applied to their extremities (resulting on a shifting of their resonance frequency). DETF resonators present the advantages of higher quality factor (increasing the device performance) and zero net force at anchors [17]. The higher quality factor is explained by the stress wave canceling in 
case of anti-phase driving of the beams. A disadvantage of DETFs in comparison to single-beam is a lower sensitivity (usually few $\mathrm{Hz} / \mathrm{g}$ ) because of an increased cross-sectional area, which is usually compensated with larger proof masses and larger device areas. An alternative to mitigate this problem, in order to benefit from the several DETF advantages, is to use force amplification mechanisms, such as levers. These increase the force applied to the edges of the transducers and consequently their sensitivity. Levers can have several configurations [18]. Besides three types of levers defined by relative positions between input, output and pivot, there are also single-stage [11] and dual-stage levers [19].

In order to electrically measure the vibration of the resonators, capacitive readouts [10] are generally employed, since they offer good noise performance and ease of fabrication. Piezoelectric sensing [19] can also be found, but it is inconvenient due to the lack of compatibility with standard silicon MEMS processes. Considering the frequency modulated devices with capacitive readouts, many instrumentation systems were demonstrated. For open-loop measurements in a lab setting, signal analyzers [9,11] are mainly employed. In contrast, for closed-loop measurements, and regarding higher integration levels, authors have been generally working on self-oscillator circuits $[6,20]$ and lock-in amplifiers with built-in phase-locked loop [21].

A small and highly sensitive MEMS accelerometer composed of DETF resonators is presented in this paper, expanding and further detailing the earlier results shown in [22] and [23]. The device has a pair of DETFs coupled to the central mass, allowing a differential measurement, doubling the sensitivity to external accelerations and reducing unwanted effects, for instance, temperature dependence. The accelerometer detailed design including the leverage mechanism for sensitivity enhancement is described in Section 2. The standard Bosch silicon surface micromachining process used for the fabrication of the microstructures is also described. In Section 3, the system implementation approaches, based on lockin amplifier instrumentation are presented. All the experimental results are shown in Section 4 and a brief conclusion is given in Section 5.

\section{Accelerometer design, optimization and fabrication}

\subsection{Device design}

The in-plane single-axis resonant accelerometer comprises an inertial mass, two levers, and two DETFs, for differential measurement (proof-mass movement yields tensile stress on one DETF and compressive on the other), as schematized in Fig. 1. Axial forces acting on the DETFs induce slight changes in the stiffness, and consequently a proportional shift on the natural frequency.

DETFs are driven to the first anti-phase resonance mode through electrostatic actuation. In addition to the driving and sensing electrodes, as shown in Fig. 1, the proposed device uses set of electrodes coupled to the center of the DETF (the deflecting beams are not used as electrodes as opposed to [11]), increasing the capacitance variation, and hence the signal-to-noise ratio, at the expense of sensitivity (the DETF mechanical sensitivity is inversely proportional to the mass of the electrodes [24]).

The resonance frequency shift $(\Delta \mathrm{f})$, i.e. the difference between the resonance frequency $(f)$ when a force $(F)$ is applied and the nominal natural frequency $\left(\mathrm{f}_{0}\right)$ (with no force applied), can be calculated using:

$$
\Delta f=\frac{1}{2 \pi}\left(\sqrt{\frac{\frac{192 \times E \times I}{L^{3}}\left(1+\frac{L^{2} \times F}{40 \times E \times I}\right)}{M_{e}+0.38 M}}-\sqrt{\frac{\frac{192 \times E \times I}{L^{3}}}{M_{e}+0.38 M}}\right)
$$

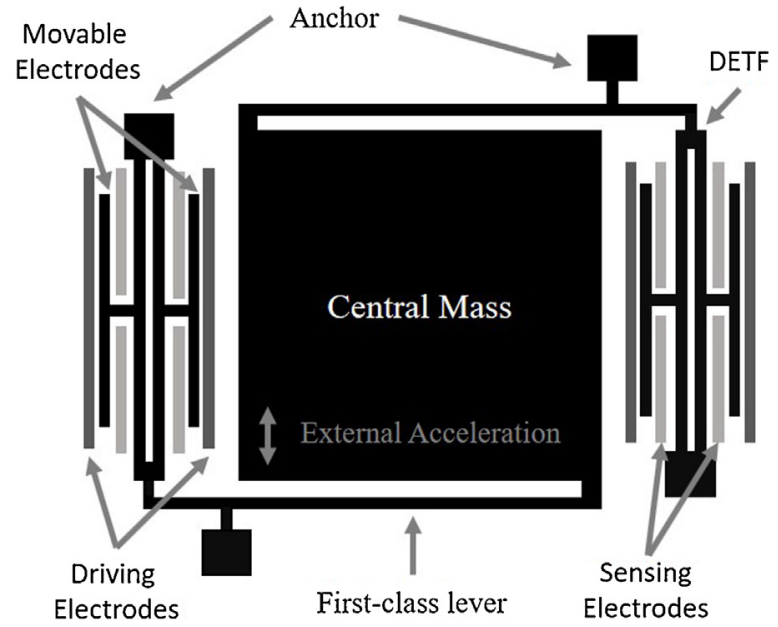

Fig. 1. Simplified schematic of the proposed differential resonant accelerometer.

where $\mathrm{L}$ and $\mathrm{M}$ are respectively the length and mass of each DETF beam, E the Young's modulus of silicon, I the second moment of area and $\mathrm{M}_{\mathrm{e}}$ the mass of the coupled electrode.

The sensitivity of this type of device is often maximized by using longer, thinner beams coupled to larger proof masses (since $\mathrm{F}=\mathrm{M}_{\text {proof }} \times \mathrm{a}_{\text {ext }}$, being $\mathrm{M}_{\text {proof }}$ the proof mass and $\mathrm{a}_{\mathrm{ext}}$ the external acceleration), which leads to devices with large dimensions. This is the main limitation on achieving highly sensitive DETF-based sensors with small footprints. The force amplification mechanism implemented to overcome this limitation is discussed in Section 2.3.

Depending on the direction of the external acceleration, the force applied to a DETF can be tensile or compressive, which leads to a positive or negative drift on the resonance frequency and enables bi-directional sensing. The proposed differential architecture doubles the sensitivity while reducing unwanted effects acting on both sides (left and right DETF) with the same amplitude, for instance, sensor drift over temperature.

\subsection{Device modelling}

The electrical connections of a single DETF are schematized in Fig. 2. The interaction between the electrical stimuli and the mechanical properties of the devices must be taken into consideration on the complete system model. The system was modeled as combination of three second-order models in Simulink (right DETF, left DETF and accelerometer central mass), including electrostatic forces acting on the DETFs (from both the drive electrodes and the sensing ones), elastic forces (on the DETF vibration direction and on the proof-mass movement direction since the lever acts as spring), inertia (on the DETF beams and on the proof-mass), squeeze-film damping on the DETFs and slide damping between the proof-mass and the substrate at $2 \mu \mathrm{m}$ (constraint from the fabrication process).

The DETF beams are excited by a sinusoidal voltage (va) producing an electrostatic force that drives the DETF at its natural frequency. In addition to the driving signal, a bias voltage $(\mathrm{Vp})$ is applied to the center of the DETF to enable capacitive sensing.

Using the dynamic model of the complete system, an optimized design was achieved. The design parameters of the complete device are given in Table 1. Naturally, the device parameters were also constrained by fabrication process limitations. The minimum beam width accepted is $1.4 \mu \mathrm{m}$ and the minimum gap $1.6 \mu \mathrm{m}$, affecting both sensitivity and quality factor. Additionally, the device footprint was limited to an area around $0.25 \mathrm{~mm}^{2}$, the active layer thickness was $19 \mu \mathrm{m}$ and the encapsulation pressure was fixed at $150 \mathrm{~Pa}$ (for compatibility with other co-encapsulated devices). 


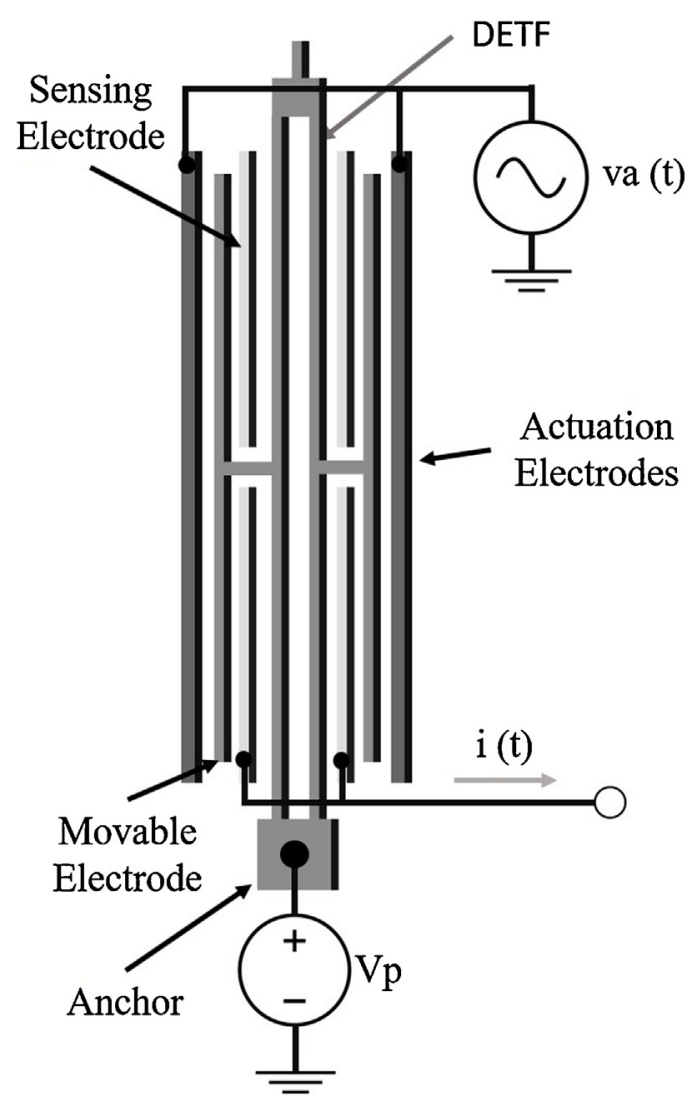

Fig. 2. Schematic of the different electrical connections and components constituting the DETF actuation and sensing elements.

Table 1

Design parameters of the device.

\begin{tabular}{lll}
\hline Parameter & Parameter description & Value \\
\hline$W s$ & DETF support width & $1.4 \mu \mathrm{m}$ \\
$L s$ & DETF support length & $10 \mu \mathrm{m}$ \\
$L a$ & Output lever length & $2.25 \mu \mathrm{m}$ \\
$W p$ & Pivot width & $1.4 \mu \mathrm{m}$ \\
$L p$ & Pivot length & $20 \mu \mathrm{m}$ \\
- & Lever length & $430 \mu \mathrm{m}$ \\
$t$ & Device thickness & $19 \mu \mathrm{m}$ \\
$L$ & DETF beam length & $400 \mu \mathrm{m}$ \\
$w$ & DETF beam width & $1.4 \mu \mathrm{m}$ \\
$L_{e}$ & Movable electrode Length & $300 \mu \mathrm{m}$ \\
$W_{e}$ & Movable electrode width & $2 \mu \mathrm{m}$ \\
$M_{\text {proof }}$ & Proof mass & $5.32 \times 10^{-9} \mathrm{Kg}$ \\
- & Proof mass natural frequency & $1.2 \mathrm{kHz}$ \\
$d_{0}$ & Rest distance (gap) & $1.6 \mu \mathrm{m}$ \\
$f_{0}$ & DETF natural frequency & $\approx 38.7 \mathrm{kHz}$ \\
$Q$ & Quality factor (DETF) & 100 \\
$P_{0}$ & Encapsulation Pressure & $150 \mathrm{~Pa}$ \\
\hline
\end{tabular}

The expected DETF quality factor is lower than what is typically observed on resonant devices. The main contribution for the low-quality factor is the squeeze-film damping between the movable and fixed electrodes which is significant at the encapsulation pressure considered (specific of the microfabrication employed).

\subsection{Sensitivity optimization}

Force amplification mechanisms have been used in many applications where force or displacement are meant to be transferred from an input to an output with a gain factor (above or below 1) [18]. In micromechanical systems, these mechanisms have been employed to increase displacement and consequently sensitivity

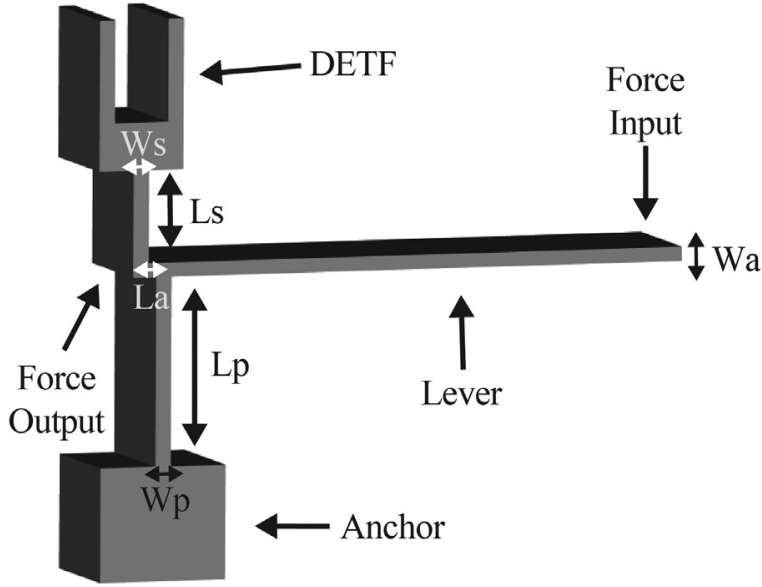

Fig. 3. Schematic representing the features and the sizes that were optimized using parametric CAD design and FEM analysis. The parts depicted are the lever (and its different components), the pivot and the connection to the DETF.

of open-loop devices based on direct displacement transduction [25] but also to increase the force induced at the extremities of resonators [16]. These capabilities have already been demonstrated, however, in resonant accelerometers, authors are now being challenged to come up with highly sensitive architectures on increasingly smaller size devices. Moreover, sensors fabricated using surface micromachining have usually thin device layers, resulting in small proof masses, further increasing the importance of these force amplification mechanisms.

In Fig. 1, it is shown that the lever used in this design is a single-stage, first-class lever with a rotational pivot, differing from the device presented in [11]. The levers' performance was studied using FEM modelling linked with a parametric CAD design of the complete structure, since no accurate analytical models can be found, especially considering that perfect pivots cannot be micromachined. This allows a straight-forward optimization of all the features' dimensions to maximize the sensitivity obtained by the lever (in this case, the lever is acting as a spring and as a force amplification mechanism). For the sensitivity/lever gain optimization, the features studied were the length of the output lever (La), the lever width (Wa) and the width and length of the pivot (Wp and Lp, respectively), see Fig. 3.

Also, the width (Ws) and the length (Ls) of the DETF connector were considered. The lever length is similarly a crucial factor, but due to size restrictions, this parameter was fixed to a maximum of $430 \mu \mathrm{m}$. The study yielded an optimal sensitivity point dependent on the length of the output lever (La), see Fig. 4, in accordance with other published studies [11]. Here, similar studies were performed for each parameter, and the results in Fig. 4 show the output lever length dependency with all other parameters already optimized. The maximum sensitivity value of $52.2 \mathrm{~Hz} / \mathrm{g}$ corresponds to a single DETF, hence a total sensitivity of $104.4 \mathrm{~Hz} / \mathrm{g}$ can theoretically be achieved with a differential configuration. This corresponds to a sensitivity improvement of around 20 times, when compared to a simple DETF without any amplification mechanism (Eq. (1)).

\subsection{MEMS fabrication}

The designed devices were fabricated in a surface micromachining process (Bosch proprietary) developed for MEMS sensors, with a polysilicon active layer of $19 \mu \mathrm{m}$ and buried electrical connections. The buried connections allow design versatility and a reduction of the total device area. The fabricated device, shown in Fig. 5, is composed of two DETF, a central perforated mass, two levers and a central mechanical stopper. 


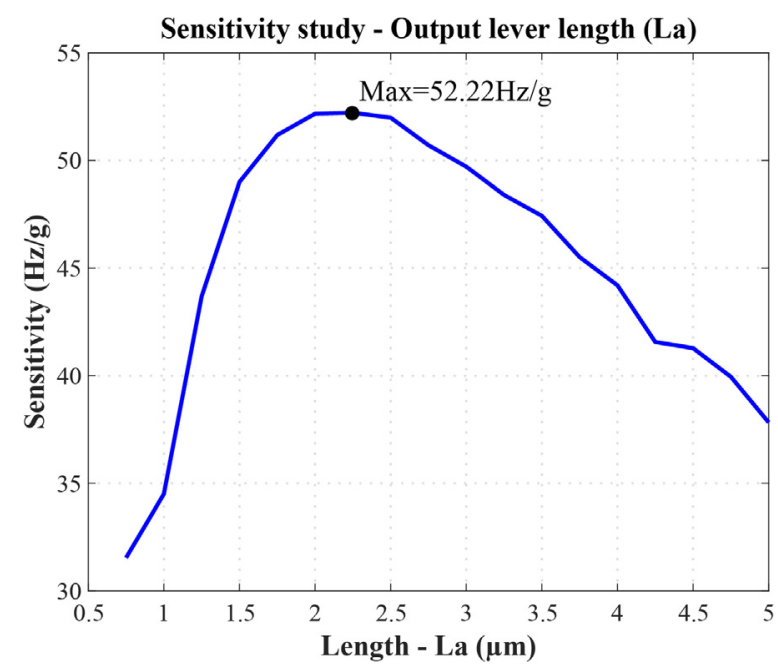

Fig. 4. Sensitivity obtained through FEM simulation linked with a parametric CAD design, showing the behavior of the system sensitivity accordingly to the length of the output lever (La). The values of the other features' dimension were fixed in this set of results.

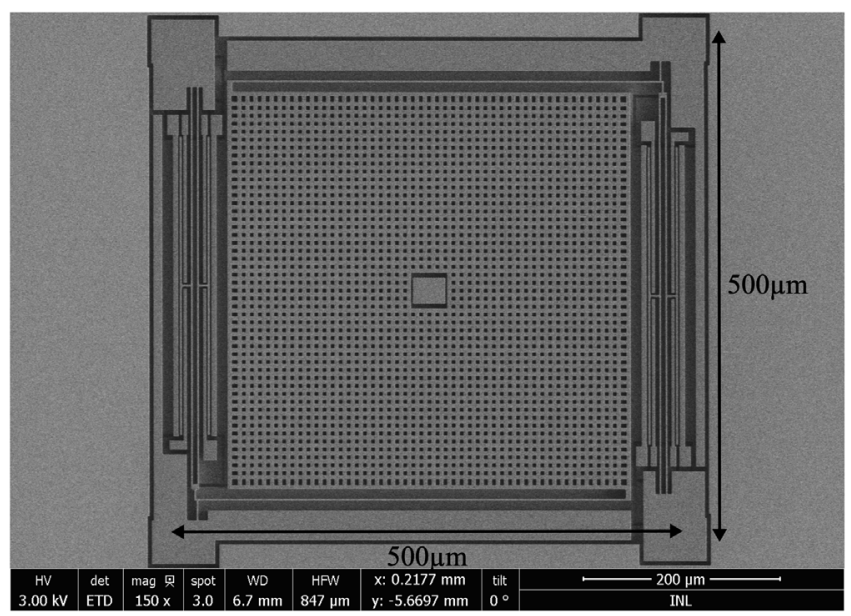

Fig. 5. SEM picture of the structure fabricated at Bosch using a micromachining process with low-pressure encapsulation.

The mechanical stopper was added to restrain the displacement of the central mass (in the $\mathrm{X}$ and $\mathrm{Y}$ axis), avoiding damage of the compliant structures when over-range accelerations are applied. The stoppers were placed at a distance corresponding to the displacement of the central mass for an acceleration of $\pm 25 \mathrm{~g}$ (dynamic range), however the measurement range of interest for the targeted applications is $\pm 5 \mathrm{~g}$, which was the range experimentally tested.

The structures were encapsulated in vacuum by wafer bonding. The quality factor of resonant accelerometers can be increased by decreasing the encapsulation pressure (at the expense of operation range). In this work, the device was designed to be operated at the pressure $150 \mathrm{~Pa}$, which is specific of the microfabrication process employed.

In addition, the silicon dies were glued to a CLCC chip carrier using a soft silicone for stress decoupling and then wire-bonded (see Fig. 6).

\section{System implementation}

In order to evaluate the static response of the resonators and perform a preliminary characterization, the DETF were operated in open-loop control, i.e. without updating the excitation frequency.

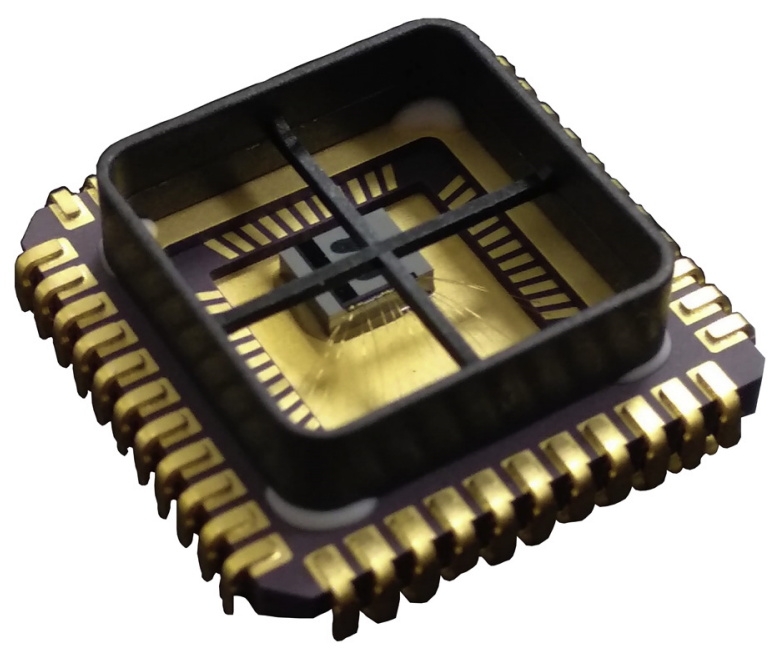

Fig. 6. Silicon die wire-bonded and additionally, glued to a PLCC chip carrier using a soft silicone.

This characterization consisted mainly on performing a frequency sweep to obtain the bode plot of the devices and determine nominal resonance frequencies and quality factors. All the remaining characterization of the device was performed in closed-loop control, since the sensor is intended for close-loop operation.

The DETF resonators' deflection is transduced to a capacitance change on the sensing electrodes. Considering that a DC bias voltage $(\mathrm{Vp})$ is used, this small capacitance change (considering the electrodes area and gap distance) generates low amplitude currents (in the $\mathrm{pA}$ and $\mathrm{nA}$ range). This highlights the importance of the movable electrodes coupled to the DETF beams highest deflection point to increase capacitance variation. The current is instrumented through a transimpedance amplifier that converts the current into voltage. A second amplification stage in an inverting configuration was added for further amplification, as demonstrated in Fig. 7. Those amplification stages were implemented using operational amplifiers from Analog Devices (AD8066).

The amplified output voltage is read by a lock-in amplifier (HF2LI from Zurich Instruments), returning information about the amplitude and phase of the signal. The lock-in amplifier allows to drive the resonators at half of their natural frequency, which is a great advantage due to decoupling of the stray capacitance. Because of the low capacitance changes on the devices, stray capacitance jeopardizes the correct signal instrumentation. Often, the signal cannot be read or exhibits a merged response between the stray and sensor capacitance, culminating on signals with anti-resonance peaks or phase shifts at their natural frequency below $90^{\circ}$ (as theoretically expected for a second-order mechanical system). To correct this issue, the driving signal applied is at half of the natural frequency of the device and the lock-in reads the second harmonic of the sensor signal. The second harmonic is at the natural frequency of the DETF. This technique is used to decouple the stray capacitance from the signal of the sensor. The phase and amplitude are monitored at $\mathrm{f}_{0}$ returning the output of the DETF.

The closed-loop system implemented (see Fig. 7) uses additional lock-in functionalities and acquisition and control subsystems (see highlighted components in Fig. 7), including an FPGA and a data acquisition board.

The analog voltage representing the phase shift of the DETF (one output of the lock-in amplifier) is converted into the digital domain through an analog-to-digital converter (ADC) and used as input to the proportional-integral-derivative (PID) controller implemented on a field-programmable gate array (FPGA) that updates the driving voltage frequency. The controller calculates the new resonance frequency to be injected into the sensor at a frequency above $4 \mathrm{kHz}$ 


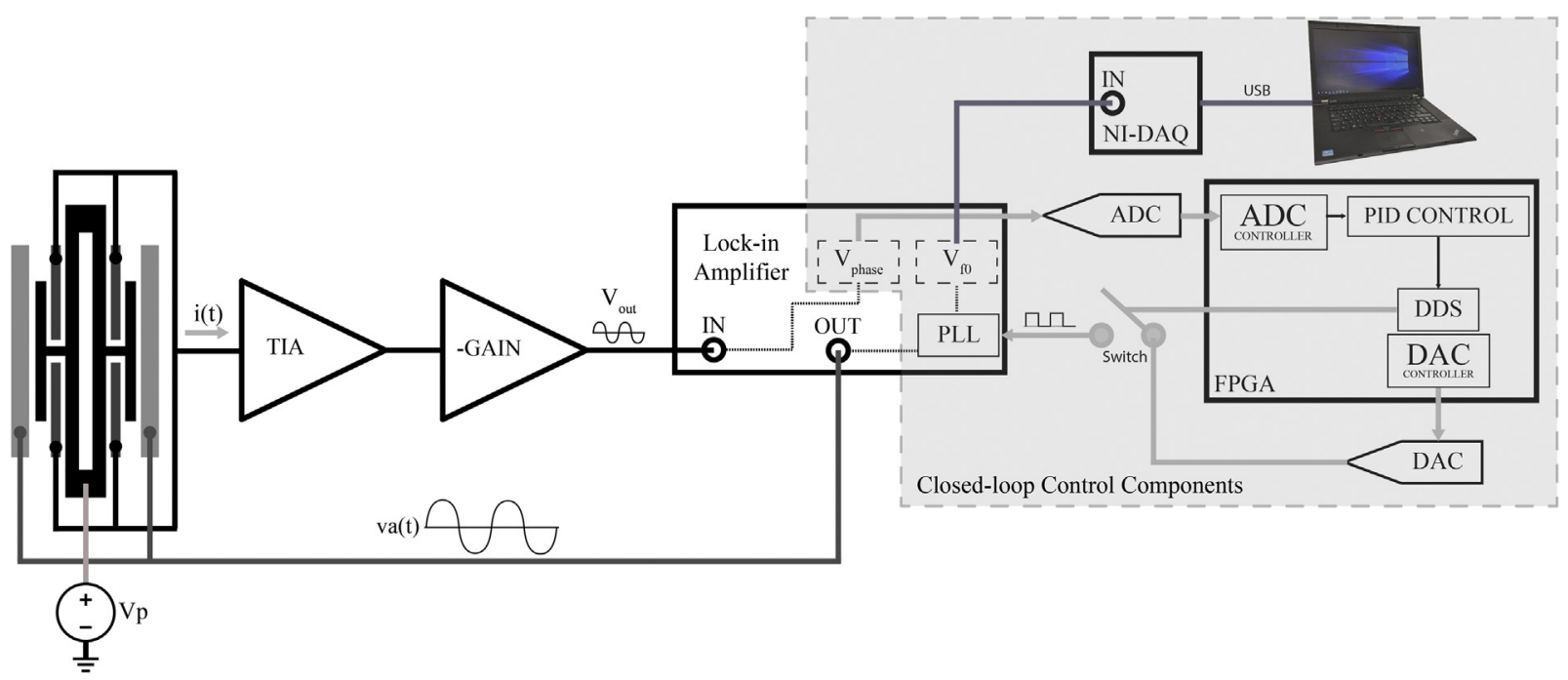

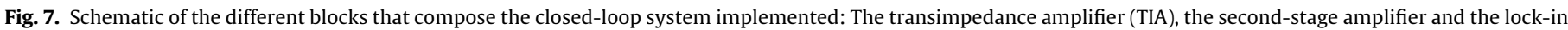

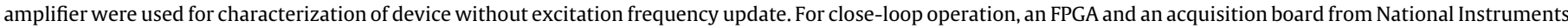
were added (highlighted, on the right). Additionally, some more functionalities of the lock-in amplifier were used to close the loop.

(frequency at which the loop is closed). To generate a square wave signal at the desired frequency, a direct digital synthesis (DDS) module was implemented.

$\Delta f_{\text {inc }}=\frac{f_{\text {clk }}}{2^{\text {nbits }}}$

This digital module frequency increment resolution $\left(\Delta \mathrm{f}_{\text {inc }}\right)$, considering an accumulator of 32 bits ( $\mathrm{n}_{\text {bits }}$ ) and a fixed clock of $50 \mathrm{MHz}$ $\left(\mathrm{f}_{\mathrm{clk}}\right)$ is expected to be $12 \mathrm{mHz}$, according to (2) [26]. The frequency increment is important, since it limits the final resolution of the sensor. The DDS controls the switching between a voltage given by a digital to analog converter (DAC) and ground, through an external switch. This results in a square wave signal at the target DETF excitation frequency. A phase-locked loop (PLL) reads the frequency of this signal controlled by the FPGA and transposes it to the output at the same signal frequency but with a different waveform. A sinewave with an amplitude va is then injected to the sensor (to eliminate exciting harmonics), thus closing the loop.

A voltage proportional to the updated excitation frequency $\left(\mathrm{V}_{\mathrm{f} 0}\right)$ (output of the lock-in) is sampled by a data acquisition board (NIDAQ) and sent to a computer through a USB connection to be used as the system output.

Fig. 7 depicts the reading and actuation setup of a single DETF. To operate the entire device, the front-end electronics was duplicated as well as the digital modules implemented on the FPGA, and two inputs and two outputs of the lock-in amplifier were used, corresponding to the entire capacity of the lock-in amplifier (two inputs, two outputs, four auxiliary outputs and two PLL). The response of the right and left DETF are subtracted, producing the differential reading.

\section{Experimental results}

\subsection{Natural frequency and quality factor}

A frequency sweep was performed in open-loop operation to extract the natural frequencies and quality factors of both DETFs, as shown in Fig. 8. A natural frequency close to $35.8 \mathrm{kHz}$ at $0 \mathrm{~g}$ was measured in both resonators, $3 \mathrm{kHz}$ below the theoretical value, representing a $7.5 \%$ difference. Furthermore, quality factors close to 130 were experimentally measured. This indicates an over-etch of $5 \%-10 \%$. The observed mismatch in natural frequency and quality
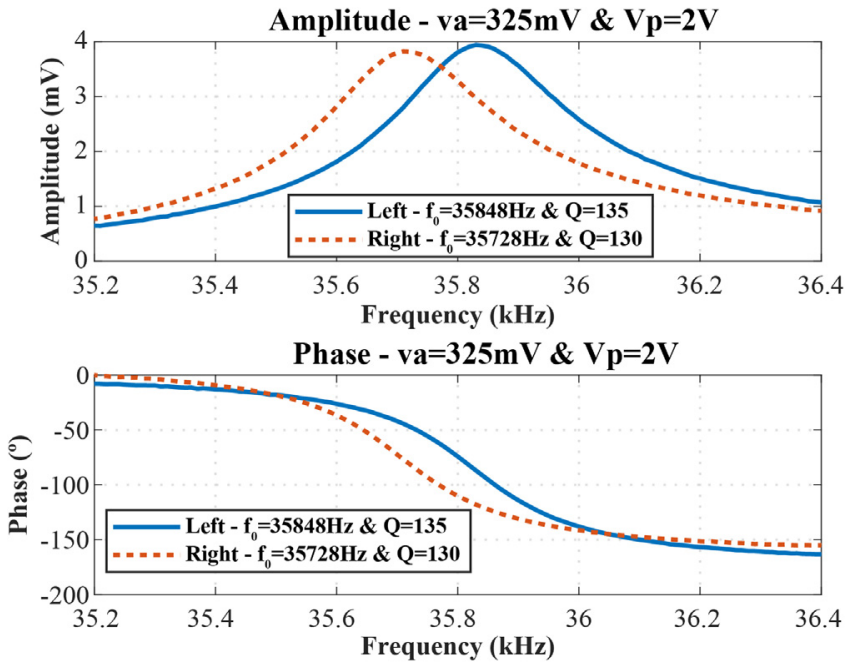

Fig. 8. Bode plot of the left and right DETFs response.

factor on the sensors can be explained by process non-uniformities which result in slight differences between left and right DETF and/or different mechanical pre-stress.

A total of five samples were experimentally measured, meaning the frequency response of ten DETFs were examined. An average natural frequency of about $35928 \mathrm{~Hz}(\sigma=417.5 \mathrm{~Hz})$ and a quality factor of $121.4(\sigma=8.59)$ were obtained for the same conditions as the measurement shown in the figure above.

\subsection{Sensitivity and linearity}

The sensors were tested in a climate chamber at fixed temperature of $25^{\circ} \mathrm{C}$, with a high precision rotational motor, as shown in Fig. 9.

The rotational motor allows increments of gravitational acceleration of few $\mathrm{mg}$. The response of the sensors was experimentally measured in the range $[-1 \mathrm{~g},+1 \mathrm{~g}]$ with increments of $10 \mathrm{mg}$ at a rate of $30 \mathrm{~s} / \mathrm{step}$ ( $1 \mathrm{kHz}$ sampling rate i.e. 30000 samples/10 mg). In Fig. 10, the sensitivity of the devices is shown. A maximum sensitivity of approximately $170.7 \mathrm{~Hz} / \mathrm{g}$ and a non-linearity of 0.63 $\%$ FS were obtained for the sample \#1. An average sensitivity of 


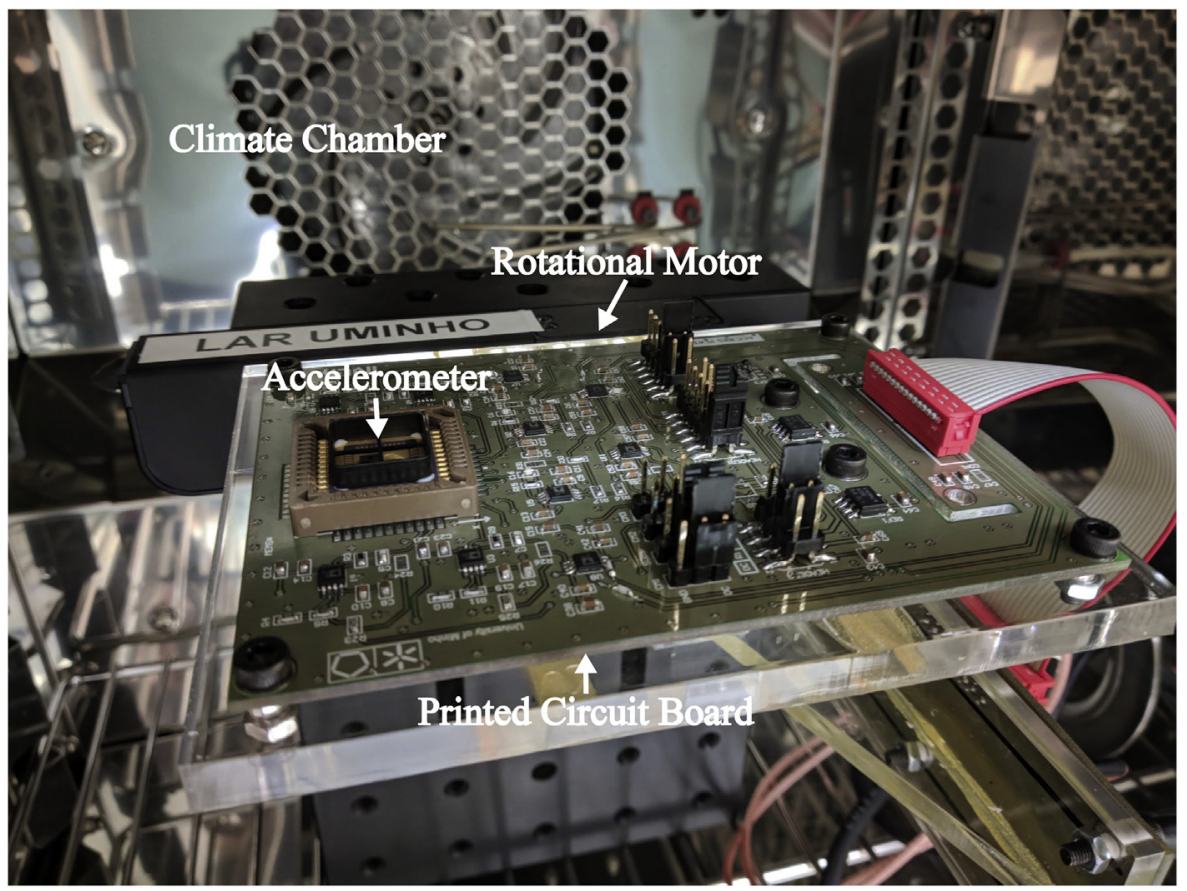

Fig. 9. Photography of the test setup inside the climate chamber.

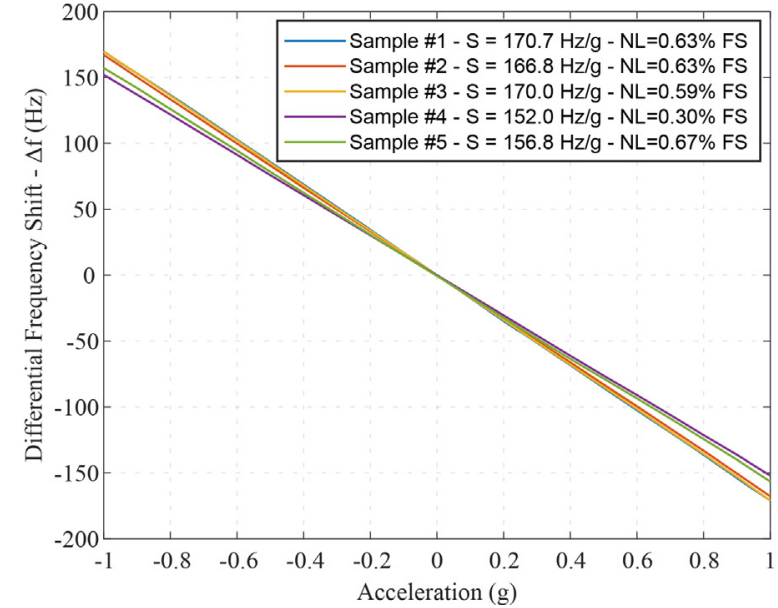

Fig. 10. Experimental sensitivity $(S)$ measured on five different samples for the same driving and bias voltages $(\mathrm{Vp}=2 \mathrm{~V}$ and $\mathrm{va}=300 \mathrm{mV})$ and the non-linearity (NL) calculated using a linear fitting.

$163.7 \mathrm{~Hz} / \mathrm{g}$ and non-linearity of $0.56 \%$ were measured on five different samples. The experimental sensitivity is well above the designed value ( $\sim 70 \%$ in the worst case). This is due to the overetch which positively affects the sensitivity, since thinner DETF beams result in higher frequency drifts, and thinner pivots and levers increase the force amplification.

In Table 2 a comparison is shown between the device reported here and highly sensitive devices reported in literature. For frequency modulated accelerometers, the frequency shift per nominal frequency per unit of acceleration $\left(\left(\Delta \mathrm{f} / \mathrm{f}_{0}\right) / \mathrm{a}_{\mathrm{ext}}\right.$, which can be displayed in $\% \mathrm{~Hz} / \mathrm{g})$ is a commonly used figure of merit. A figure of merit in terms of $\left[(\Delta \mathrm{f} / \mathrm{f}) / \mathrm{a}_{\text {ext }}\right] / \mathrm{M}_{\text {proof }}$ has also been defined (in units of $\% \mathrm{~Hz} / \mathrm{g} / \mathrm{nkg}$ - nkg being used instead of $\mu \mathrm{g}$ as to prevent misreading as acceleration), as it reflects the efficiency of the transduction mechanism irrespectively to the mass size. As shown in Table 2, regarding these figures of merit, or relative sensitivities, one can observe that the device developed presents one of the highest per- formance reported. In fact, the only device presenting a higher relative sensitivity per proof mass $(\% \mathrm{~Hz} / \mathrm{g} / \mathrm{nkg})$ is the one reported in [11], which concerns a clamped-clamped resonator on a differential configuration using a force amplification mechanism. This highlights the benefit of using these amplification mechanisms like the one proposed in this work. The performance experimentally measured here is further highlighted when comparing with devices composed of the same type of resonators (DETF).

\subsection{Dynamic response}

For illustrative purposes, and from the sensitivity results presented in Fig. 10, the best performing device (sample \#1) was used for the remaining experiments. The device was tested on a vibrational platform, where its dynamic response was characterized. An acceleration frequency sweep was performed from $20 \mathrm{~Hz}$ to $300 \mathrm{~Hz}$ with acceleration amplitudes [ $-1 \mathrm{~g}+1 \mathrm{~g}]$ and a bandwidth (BW) of $63 \mathrm{~Hz}$ was experimentally measured (see Fig. 11). In open-loop, the theoretical bandwidth $\left(B W=f_{0} / 2 Q\right.$ ) is expected to be close to $137 \mathrm{~Hz}$. The overall accelerometer system BW will be limited mechanically by the component with the smallest BW (the DETF resonators or the proof-mass system) and electronically by the BW of the control loop. In the case of this device, the DETF BW is lower than the proof mass system's BW (approximately $1.2 \mathrm{kHz}$ ), so the theoretical BW (due to mechanical factors, i.e. not accounting for control loop performance) was calculated based on the DETF resonator parameters. The lower $63 \mathrm{~Hz}$ bandwidth experimentally measured is believed to be the result of limitations on the closedloop approach using a PLL and a PID controller implemented on the FPGA. In fact, the different PLL parameters were manually tuned to increase this figure. Experimentally, it was not possible to increase it further than $63 \mathrm{~Hz}$ (beyond the initial $10 \mathrm{~Hz}$ obtained using the PLL default configuration). Additional work needs to be performed to improve this limitation, namely, integrating the lock-in amplifier on the FPGA control system.

The performance of the shaker used during the structure characterization presented an artefact around $60 \mathrm{~Hz}$, which was monitored by the reference and the designed accelerometer. A 
Table 2

Sensitivity of accelerometers based on several resonators' types (clamped-clamped beam, fishbone resonator and DETF).

\begin{tabular}{|c|c|c|c|c|c|c|c|}
\hline \multirow[t]{2}{*}{ Resonator Type } & \multirow[t]{2}{*}{ Work } & \multirow[t]{2}{*}{ Proof Mass (nkg) } & \multirow[t]{2}{*}{ Natural Freq.(kHz) } & \multirow[t]{2}{*}{ Scale factor $(\mathrm{Hz} / \mathrm{g})$} & \multirow[t]{2}{*}{ Die area $\left(\mathrm{mm}^{2}\right)$} & \multicolumn{2}{|c|}{ Relative Sensitivity } \\
\hline & & & & & & $\frac{\Delta f / f_{0}}{a_{e x t}}(\% \mathrm{~Hz} / \mathrm{g})$ & 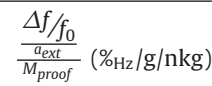 \\
\hline \multirow{5}{*}{$\begin{array}{l}\text { Clamped-clamped } \\
\text { beam } \\
\text { Fishbone resonator }\end{array}$} & [27] & $0.098^{a}$ & 459.15 & 22 & 0.05 & 0.0048 & 0.0489 \\
\hline & [11] & $3.55^{\mathrm{a}}$ & 58 & 455 & $0.16^{\mathrm{b}}$ & 0.78 & 0.22 \\
\hline & [28] & $71.2^{\mathrm{a}}$ & $547.2^{\mathrm{d}}$ & $61^{\mathrm{d}}$ & $1.2^{\mathrm{b}}$ & 0.01 & 0.0001 \\
\hline & [3] & 309 & 25.238 & 31.56 & 9.92 & 0.13 & 0.0004 \\
\hline & [19] & $23.5^{\mathrm{a}}$ & 140.7 & 28.4 & 3.2 & 0.02 & 0.0009 \\
\hline \multirow{5}{*}{ DETF } & [29] & 557.1 & 350 & 1400 & NA & 0.4 & 0.0007 \\
\hline & [30] & $32.2^{\mathrm{a}}$ & 130 & 158 & $0.46^{\mathrm{b}}$ & 0.12 & 0.0038 \\
\hline & [16] & 1.33 & 173 & 17 & NA & 0.01 & 0.0074 \\
\hline & [31] & $2444^{a}$ & 149.52 & 9408 & $26^{c}$ & 6.29 & 0.0026 \\
\hline & This work & 5.32 & 35.8 & 170.7 & 0.25 & 0.48 & 0.0895 \\
\hline
\end{tabular}

The bold values are meant to enhance the best values of the state-of-the-art.

a Mass calculated using a silicon density of $2350 \mathrm{~kg} / \mathrm{m}^{3}$ NA- Not available.

b Only the area of the mass is available.

c Only the volume of the mass is available.

d The third mode was considered.
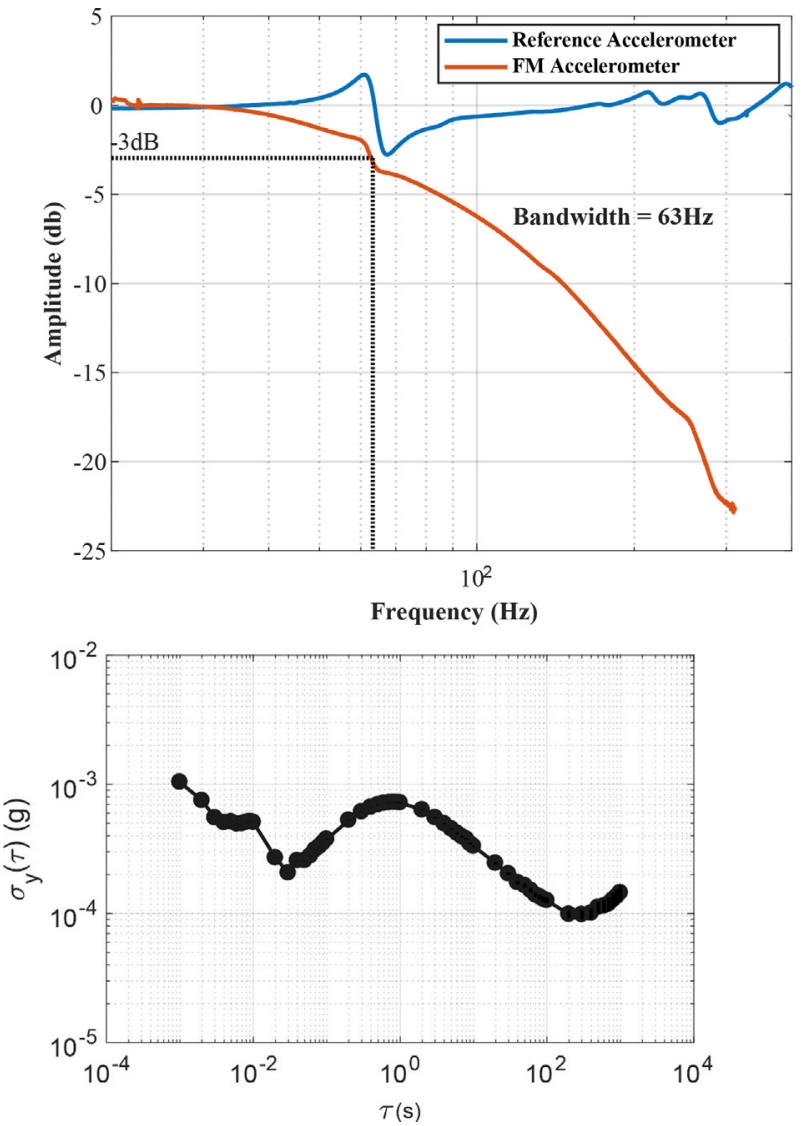

Fig. 11. Bandwidth experimentally measured on the shaker with $\pm 1 \mathrm{~g}$ excitation, the natural frequency shift is presented in $\mathrm{dB}$ for the sake of comparison with the reference accelerometer used for result validation.

slight misalignment may have resulted in a smaller peak amplitude applied to the frequency-modulated (FM) accelerometer.

The proof mass natural frequency is expected to be approximately $1.2 \mathrm{kHz}$. Since it is well above the bandwidth of the system, it is expected that it is not limiting the system's bandwidth.

To evaluate the closed-loop dynamic response, the acceleration was incremented from $\pm 0.1 \mathrm{~g}$ to $\pm 5 \mathrm{~g}$ with steps of $0.1 \mathrm{~g}$, at $20 \mathrm{~Hz}$. The sensor response, translated using the previously measured scale factor, is shown in Fig. 12. Additionally, the figure shows a stable behavior up to the maximum acceleration tested, $\pm 5 \mathrm{~g}$.

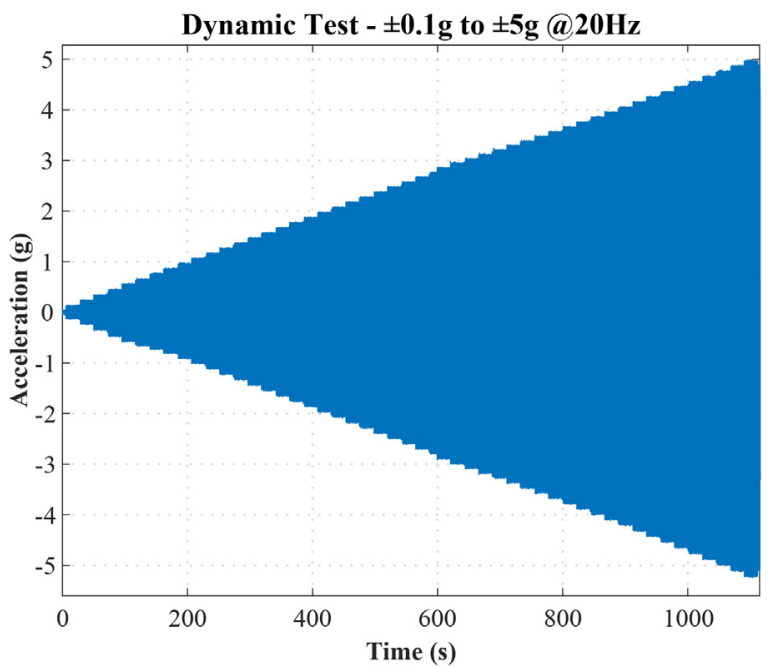

Fig. 12. Dynamic response of the device from $\pm 0.1 \mathrm{~g}$ to $\pm 5 \mathrm{~g}$ at $20 \mathrm{~Hz}$. The acceleration in the yy axis is the value measured by the frequency modulated accelerometer.

\subsection{Temperature dependency}

The device was tested inside the climate chamber at $0 \mathrm{~g}$ and the temperature was increased from approximately $30^{\circ} \mathrm{C}-70^{\circ} \mathrm{C}$. The resonance frequency shift of the resonators was measured, as shown in Fig. 13. It is shown that the resonance frequency of both DETFs increases with temperature, but at different rates. Theoretically, both sensors (left and right) should have the same behavior at different temperatures, although some differences are expected to arise from fabrication process non-uniformities. Although the frequency response of resonators should decrease with temperature (Young's modulus decreases with temperature), the effect measured was in the opposite direction. This is explained by the introduction of tensile stress on the DETF through the anchors, which results from the thermal expansion of the packaging, the main contribution for this effect. Both the magnitude of the temperature drift and the mismatch between the two DETF results were higher than expected, due to this stress coupling. Nevertheless, it is shown that the differential measurement can be used to reduce the response drift of the device from $8.7 \mathrm{~Hz} /{ }^{\circ} \mathrm{C}$ (worst case) to $2 \mathrm{~Hz} /{ }^{\circ} \mathrm{C}$. To improve the poor thermal behavior, several solutions can be implemented in future work: the use of an improved packaging method for stress decoupling [32], where the die is glued to a stressimmune auxiliary structure; reducing the die attachment area to a 


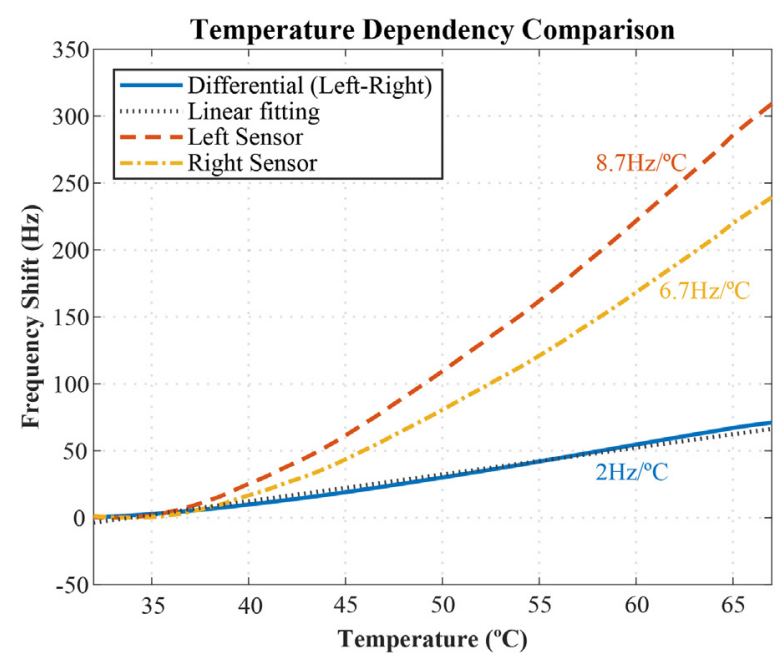

Fig. 13. Temperature dependency of the left and right DETF, and additionally, the temperature dependency of the differential approach.

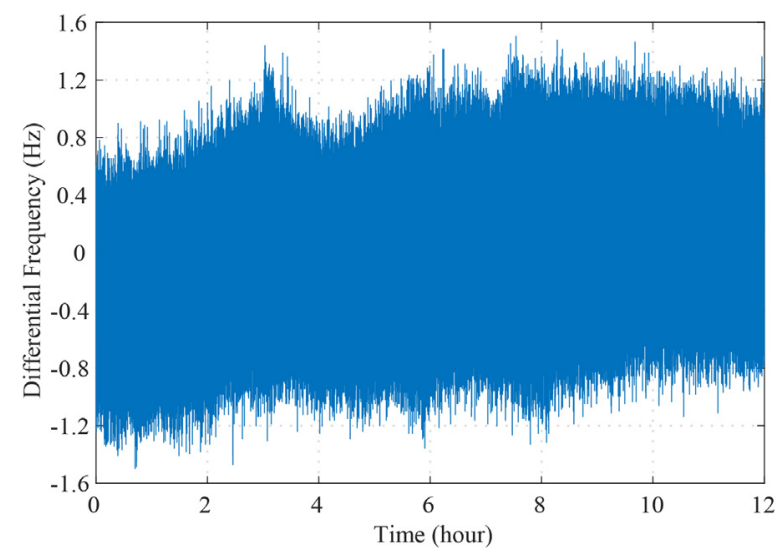

Fig. 14. Long-term measurement $(12 \mathrm{~h})$ of the differential sensor response at $0 \mathrm{~g}$ and an amplified image of a shorter period of time. A sampling frequency of $1 \mathrm{kHz}$ was used.

minimum at the corner of the die; and/or changing the design of the structure so that both DETFs share the same anchor point [33].

\subsection{Long-term measurement}

The differential frequency shift was measured over a time period of $12 \mathrm{~h}$ on a low noise setup site. The temperature was controlled and stabilized around $22.6^{\circ} \mathrm{C}$ (to avoid bias instability related to the poor temperature behavior of the system) and the device was kept at $0 \mathrm{~g}$. The data shown in Fig. 14 shows the differential response over time in $\mathrm{Hz}$ and its noise floor was calculated. A value of $700 \mu \mathrm{g} / \sqrt{ } \mathrm{Hz}$ (limit set by the electronic noise) was experimentally measured using the closed-loop approach.

In Fig. 15, the Allan variance for the measurement performed for a period of $12 \mathrm{~h}$ is demonstrated. An unexpectedly elevated noise around $1 \mathrm{~Hz}$ is observed (away from the typical Allan Variance curves dominated at low frequencies by $1 / \mathrm{f}$ noise and by Brownian noise at high frequencies). This is believed to be due to the low level of integration of this system, which largely increases the number of possible noise sources, namely the COTS elements used and the subsystem interfaces. Further work is necessary to identify the sources of noise and improve the noise performance. Namely, a higher level of integration (implementing the lock-in amplifier on a FPGA for better control) or the use of a different instrumentation

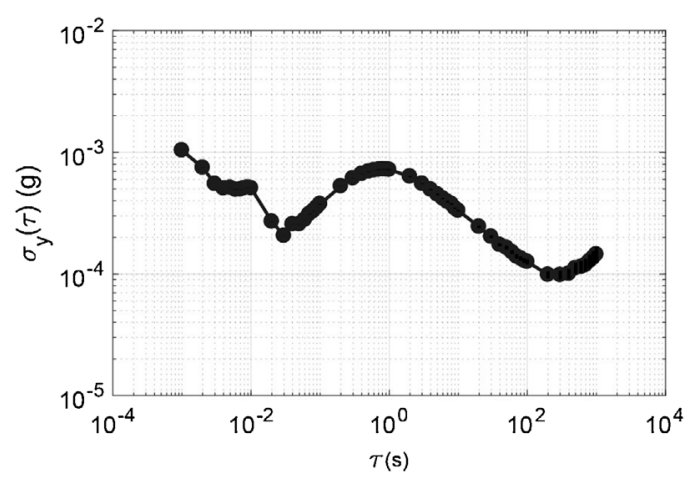

Fig. 15. Allan variance measured operating the double-ended tuning fork resonators in closed-loop for a $12 \mathrm{~h}$ period.

topology (e.g. the use of an oscillator circuit) could be approaches to mitigate these limitations.

\section{Conclusions}

A single-axis frequency modulated MEMS resonant accelerometer using a differential DETF architecture has been presented. Design and optimization were performed with the aim of increasing the sensitivity within a set of constraints such as: a) a small footprint, namely $0.25 \mathrm{~mm}^{2}$ maximum, b) operation at a vacuum of $150 \mathrm{~Pa}$ to allow co-encapsulation with other sensors typically composing an IMU, and c) for fabrication using a Bosch proprietary fabrication process with its own set of design rules. Open-loop and closed-loop results are presented and are in accordance with the expected from the models used.

The force amplification mechanism was fully optimized through FEM modelling to increase the sensitivity, with a value of $170 \mathrm{~Hz} / \mathrm{g}$ having been achieved. The experimental performance of the device was compared with other state-of-the art DETF-based structures, confirming its high sensitivity. While there are higher absolute sensitivities $\left(\Delta \mathrm{f} / \mathrm{a}_{\text {ext }}\right)$ reported, if one compares the figure of merit of the transduction mechanism independently of the proofmass used, the sensor showed the highest reported value of 0.08 $\% \mathrm{~Hz} / \mathrm{g} / \mathrm{nkg}$ for devices composed of DETF (meaning that implementing the presented architecture on larger areas or using larger proof-masses will increase the sensitivity proportionally).

A closed-loop system bandwidth of $63 \mathrm{~Hz}$ was experimentally measured. Additionally, the sensors were tested in a climate chamber at different temperatures for an extended time. The temperature dependency decreased by $77 \%$ using the differential measurement, resulting in a temperature drift of $2 \mathrm{~Hz} /{ }^{\circ} \mathrm{C}$. This value is well above the expected and is attributed to thermal stress transmitted from the package, which will be addressed in a future design by using the same anchor point for both DETFs and improving stress decoupling. Furthermore, a noise floor of about $700 \mu \mathrm{g} / \sqrt{ } \mathrm{Hz}$ was obtained for a time window of $12 \mathrm{~h}$. Future work involves developing integrated electronics and decreasing the temperature dependency.

\section{CRediT authorship contribution statement}

Eurico Esteves Moreira: Conceptualization, Methodology, Validation, Investigation, Software, Writing - original draft. Burkhard Kuhlmann: Supervision, Formal analysis, Resources, Writing review \& editing. Filipe Serra Alves: Conceptualization, Formal analysis, Writing - review \& editing. Rosana Alves Dias: Formal analysis, Writing - review \& editing. Jorge Cabral: Supervision, Resources. João Gaspar: Supervision, Resources. Luis Alexandre 
Rocha: Supervision, Conceptualization, Resources, Project administration.

\section{Declaration of Competing Interest}

The authors declare that they have no known competing financial interests or personal relationships that could have appeared to influence the work reported in this paper.

\section{Acknowledgments}

The authors would like to offer special thanks to the author Luis A. Rocha, who, although no longer with us, continues to inspire by his example and dedication to the students and collaborators he served over the course of his career.

The first author is supported by FCT- Fundação para a Ciência e Tecnologia through the grant PDE/BDE/114564/2016. This work is supported by FCT with the reference project UID/EEA/04436/2019.

\section{Appendix A. Supplementary data}

Supplementary material related to this article can be found, in the online version, at doi:https://doi.org/10.1016/j.sna.2020. 112005 .

\section{References}

[1] M. Kraft, N.M. White, MEMS for Automotive and Aerospace Applications, Woodhead Publishing Limited, Philadelphia, USA, 2013, http://dx.doi.org/10. $1533 / 9780857096487$.

[2] A. Ya'akobovitz, S. Krylov, Toward sensitivity enhancement of MEMS accelerometers using mechanical amplification mechanism, IEEE Sens. J. 10 (2010) 1311-1319, http://dx.doi.org/10.1109/JSEN.2009.2039751.

[3] J. Zhang, Y. Wang, V. Zega, Y. Su, A. Corigliano, Nonlinear dynamics under varying temperature conditions of the resonating beams of a differential resonant accelerometer, J. Micromech. Microeng. 28 (2018) 1-10, http://dx. doi.org/10.1088/1361-6439/aab7c6.

[4] S. Wang, X. Wei, Y. Weng, Y. Zhao, Z. Jiang, A novel single-axis MEMS tilt sensor with a high sensitivity in the measurement range from 0 。 to 360 。, Sensors 18 (2018) 346, http://dx.doi.org/10.3390/s18020346.

[5] A. Merdassi, M.N. Kezzo, G. Xereas, V.P. Chodavarapu, Wafer level vacuum encapsulated tri-axial accelerometer with low cross-axis sensitivity in a commercial MEMS process, Sens. Actuators A Phys. 236 (2015) 25-37, http:// dx.doi.org/10.1016/j.sna.2015.10.014.

[6] G. Laghi, C.R. Marra, P. Minotti, A. Tocchio, G. Langfelder, A 3-D micromechanical multi-loop magnetometer driven off-resonance by an on-chip resonator, J. Microelectromech. Syst. 25 (2016) 637-651, http://dx. doi.org/10.1109/JMEMS.2016.2563180.

[7] V. Lima, J. Cabral, B. Kuhlmann, L.A. Rocha, Low-pressure small MEMS accelerometer using sigma-delta modulation, Proceedings 2 (2018) 1029 http://dx.doi.org/10.3390/proceedings2131029.

[8] H. Xu, X. Liu, L. Yin, A closed-loop interface for a sigma-delta high-q micromechanical capacitive accelerometer with $200 \mathrm{ng} / \mathrm{sqrt}(\mathrm{Hz})$ input noise density, IEEE J. Solid-State Circuits 50 (2015) 2101-2112, http://dx.doi.org/10. 1109/JSSC.2015.2428278.

[9] H. Zhang, B. Li, W. Yuan, M. Kraft, H. Chang, An acceleration sensing method based on the mode localization of weakly coupled resonators, J. Microelectromech. Syst. 25 (2016) 286-296, http://dx.doi.org/10.1109/ JMEMS.2015.2514092.

[10] A. Caspani, C. Comi, A. Corigliano, G. Langfelder, A. Tocchio, Compact biaxial micromachined resonant accelerometer, J. Micromech. Microeng. 23 (2013), 105012, http://dx.doi.org/10.1088/0960-1317/23/10/105012.

[11] C. Comi, A. Corigliano, G. Langfelder, A. Longoni, A. Tocchio, B. Simoni, A resonant microaccelerometer with high sensitivity operating in an oscillating circuit, J. Microelectromech. Syst. 19 (2010) 1140-1152, http://dx.doi.org/10 1109/JMEMS.2010.2067437.

[12] A. Caspani, C. Comi, A. Corigliano, G. Langfelder, V. Zega, S. Zerbini, A differential resonant micro accelerometer for out-of-plane measurements, Procedia Eng. 87 (2014) 640-643, http://dx.doi.org/10.1016/j.proeng.2014.11 569.

[13] V. Zega, P. Minotti, G. Mussi, A. Tocchio, L. Falorni, S. Facchinetti, A. Bonfanti, A.L. Lacaita, C. Comi, G. Langfelder, A. Corigliano, The first frequency-modulated (FM) pitch gyroscope, Proceedings 1 (2017) 393, http:// dx.doi.org/10.3390/proceedings1040393.
[14] E.E. Moreira, J. Gaspar, L.A. Rocha, Frequency modulated magnetometer using a double-ended tuning fork resonator, Proceedings (2018) 1028, http://dx.doi. org/10.3390/proceedings2131028.

[15] J.E.-Y. Lee, B. Bahreyni, A.A. Seshia, An axial strain modulated double-ended tuning fork electrometer, Sens. Actuators A Phys. 148 (2008) 395-400, http:// dx.doi.org/10.1016/j.sna.2008.09.010.

[16] A.A. Seshia, M. Palaniapan, T.A. Roessig, R.T. Howe, R.W. Gooch, T.R. Schimert, $\mathrm{S}$. Montague, A vacuum packaged surface micromachined resonant accelerometer, J. Microelectromech. Syst. 11 (2002) 784-793, http://dx.doi. org/10.1109/JMEMS.2002.805207.

[17] V. Kaajakari, Practical MEMS: Design of Microsystems, Accelerometers, Gyroscopes, RF MEMS, Optical MEMS, and Microfluidic Systems, NV Small Gear Publ., Las Vegas, 2009.

[18] S.X.-P. Su, H.S. Yang, Analytical modeling and FEM Simulations of single-stage microleverage mechanism, Int. J. Mech. Sci. 44 (2002) 2217-2238, http://dx. doi.org/10.1016/S0020-7403(02)00162-5.

[19] Y. Wang, H. Ding, X. Le, W. Wang, J. Xie, A MEMS piezoelectric in-plane resonant accelerometer based on aluminum nitride with two-stage microleverage mechanism, Sens. Actuators A Phys. 254 (2017) 126-133, http://dx.doi.org/10.1016/j.sna.2016.12.019.

[20] S. Seok, H. Kim, K. Chun, An inertial-grade laterally-driven MEMS differential resonant accelerometer, Technology (2004) 654-657.

[21] M. Pandit, C. Zhao, G. Sobreviela, X. Zou, A.A. Seshia, Seismic recording using a Mode localized MEMS accelerometer, in: 20th Int. Conf. Solid-State Sensors, Actuators Microsystems Eurosensors XXXIII (TRANSDUCERS EUROSENSORS XXXIII), IEEE, 2019, pp. 2150-2153, http://dx.doi.org/10.1109/TRANSDUCERS. 2019.8808572

[22] E.E. Moreira, B. Kuhlmann, J. Gaspar, L.A. Rocha, Small size and highly sensitive differential MEMS accelerometer based on double-ended tuning fork resonators, in: 20th Int. Conf. Solid-State Sensors, Actuators Microsystems Eurosensors XXXIII (TRANSDUCERS EUROSENSORS XXXIII), IEEE, 2019, pp. 602-605, http://dx.doi.org/10.1109/TRANSDUCERS.2019.8808174.

[23] E.E. Moreira, B. Kuhlmann, J. Gaspar, L.A. Rocha, Resonant accelerometer based on double-ended tuning fork and a force amplification mechanism, Proceedings (2018), http://dx.doi.org/10.3390/proceedings2131030, p. 1030.

[24] K.E. Wojciechowski, Electronics for Resonant Sensors, University of California, Berkeley, 2005

[25] I. Zeimpekis, I. Sari, M. Kraft, Characterization of a mechanical motion amplifier applied to a MEMS accelerometer, J. Microelectromech. Syst. 21 (2012) 1032-1042, http://dx.doi.org/10.1109/JMEMS.2012.2196491.

[26] Xilinx, DDS v5.0 - Product Specification, 2005, pp. 1-26 https://www.xilinx. $\mathrm{com} /$ support/documentation/ip_documentation/dds.pdf.

[27] D. Pinto, D. Mercier, C. Kharrat, E. Colinet, V. Nguyen, B. Reig, S. Hentz, A small and high sensitivity resonant accelerometer, Procedia Chem. 1 (2009) 536-539, http://dx.doi.org/10.1016/j.proche.2009.07.134.

[28] H. Ding, W. Wang, B.-F. Ju, J. Xie, A MEMS resonant accelerometer with sensitivity enhancement and adjustment mechanisms, J. Micromech. Microeng. 27 (2017), 115010, http://dx.doi.org/10.1088/1361-6439/aa8d99.

[29] P. Xudong Zou, A.A. Thiruvenkatanathan, Seshia, A seismic-grade resonant MEMS accelerometer, J. Microelectromech. Syst. 23 (2014) 768-770, http:// dx.doi.org/10.1109/JMEMS.2014.2319196.

[30] S.X.P. Su, H.S. Yang, A.M. Agogino, A resonant accelerometer with two-stage microleverage mechanisms fabricated by SOI-MEMS technology, IEEE Sens. J. 5 (2005) 1214-1222, http://dx.doi.org/10.1109/JSEN.2005.857876.

[31] X. Zou, A.A. Seshia, A high-resolution resonant MEMS accelerometer, in: 18th Int. Conf. Solid-State Sensors, Actuators Microsystems, IEEE, 2015, pp. 1247-1250, http://dx.doi.org/10.1109/TRANSDUCERS.2015.7181156.

[32] Y. Yin, Z. Fang, Y. Liu, F. Han, Temperature-insensitive structure design of micromachined resonant accelerometers, Sensors 19 (2019) 1544, http://dx doi.org/10.3390/s19071544.

[33] D.D. Shin, Y. Chen, I.B. Flader, T.W. Kenny, Epitaxially encapsulated resonant accelerometer with an on-chip micro-oven, TRANSDUCERS 2017 - 19th Int. Conf. Solid-State Sensors, Actuators Microsystems (2017) 595-598, http://dx. doi.org/10.1109/TRANSDUCERS.2017.7994119.

\section{Biographies}

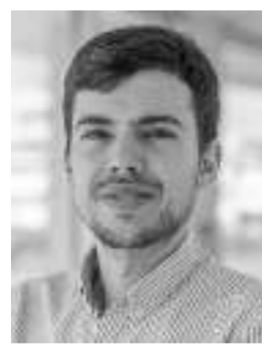

Eurico Esteves Moreira received his BSc and M.Sc. degree in Electronic Engineering from the University of Minho, Portugal, in 2012 and 2014 respectively. Currently, he is a $\mathrm{Ph} . \mathrm{D}$. student at the same university where he graduated His main focus has been on MEMS sensors and embedded systems. 


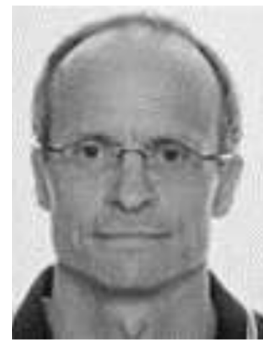

Burkhard Kuhlmann received the M.Sc. degree from the State University of New York at Buffalo in 1993, the Diploma degree in electrical engineering from the Technical University of Darmstadt, Germany, in 1994, and the Ph.D. degree in electrical engineering from the University of Hannover, Germany, in 1998. Since 1998, he has been working on MEMS inertial sensors at Robert Bosch GmbH, Reutlingen, Germany

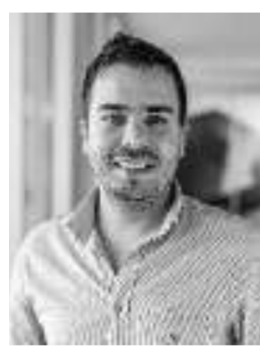

Filipe Serra Alves was born in Valença, Portugal, in 1989. He received the Ph.D. degree from the University of Minho, Portugal, in the microelectronics research field with a focus on pull-in based MEMS inclinometers with integrated electronics. He was an Assistant Professor with the Industrial Electronics Department, University of Minho. He has been a Scientific Visitor with the Delft University of Technology, The Netherlands and the International Iberian Nanotechnology Laboratory (INL). He is currently a Research Fellow with the Nanoelectronics Engineering Department, Nanodevices Research Group, INL. His research is focused on the development of integrated inter-ocular eye pressure monitoring systems, based on MEMS pressure sensors. His research interest includes the design and modeling of MEMS sensors and mixed-signal integrated circuits design.

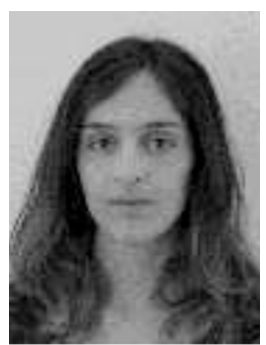

Rosana A. Dias is a Staff Researcher in the Microfabrication and Exploratory Nanotechnology research group at INL. Her expertise concerns the development of sensors and actuators to meet the technical challenges faced by society and industry. She obtained her PhD from the University of Minho in 2013, in the MEMS area, focusing on pull-in time accelerometers, inclinometers and gas viscosity sensing, at the University of Minho. Rosana has spent several months as a visitor at the Delft University of Technology and the University of British Columbia. She has also been the main responsible, as a post-doctoral researcher, for the design of a MEMS magnetometer for space applications, within a European Space Agency project. She has large experience in modelling, design, fabrication and testing of MEMS devices. Her research interests range from mechanical sensors (e.g. acceleration and magnetic field sensing) to energy harvesting devices (piezoelectric AlN and EMF-based induction), flexible substrate-based applications and optical MEMS.

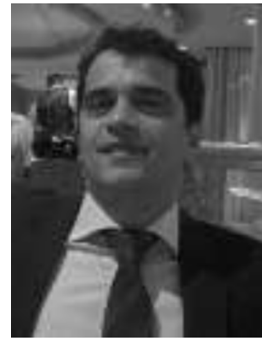

Jorge Cabral received the Ph.D. degree in microsystems technology from Imperial College London, London, U.K. He is currently an Assistant Professor with the University of Minho, Portugal. His research interests focus on embedded systems applications and he is in charge of several research projects in this field.

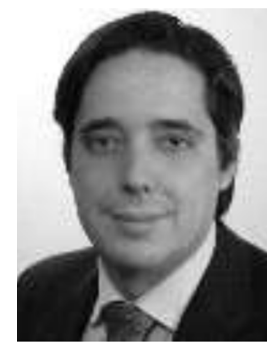

João Gaspar received the Ph.D. degree in materials engineering from the INESC Microsystems and nanotechnologies/Instituto Superior Tecnico (IST), Lisbon, in 2004 where he focused on thin-film silicon MEMS. He joined the Microsystems Materials Laboratory (MML), Department of Microsystems Engineering (IMTEK), University of Freiburg, Germany, in 2004, as a Postdoctoral Fellow for two years. In 2006, he was promoted to Lecturer and Group Leader with MML, IMTEK, where he held a position until joining the International Iberian Nanotechnology Laboratory (INL), Braga, in 2011. He is an INL Coordinator for nanofabrication and processes, and an Invited Auxiliary Professor with the Physics Department, IST. His research activities include the microfabrication processes and advanced silicon micromachining, MPW runs, high-throughput wafer-scale testing and reliability of MEMS, resonance and optical applications, microenergy harvesting, and microneedles/capillary arrays for intracellular recording plus post-CMOS processing.

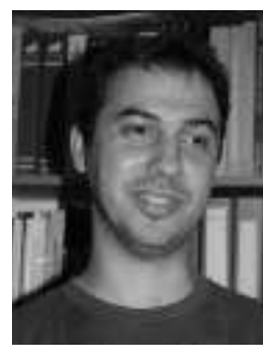

Luis Alexandre Rocha received the degree in electronics engineering from the University of Minho, in 2000, and the Ph.D. (cum laude) degree from the Delft University of Technology, in 2005. He has been a MEMS Engineer with ASML, The Netherlands, in 2005, and was an Assistant Professor with the Faculty of Engineering, University of Porto, from 2006 to 2008 . Since 2008, he has been with the University of Minho, as an Associate Professor. His research activities focus on the fabrication of microsensors based on polymer microtechnologies, and on the study and use of the nonlinear MEMS dynamic behavior for new sensor applications. 